March 8, 2019 2:34 WSPC/INSTRUCTION FILE DensityIJFCS

International Journal of Foundations of Computer Science

(C) World Scientific Publishing Company

\title{
On the Density of Context-Free and Counter Languages*
}

\author{
Joey Eremondi \\ Department of Computer Science \\ University of British Columbia, Vancouver, BC V6T 1Z4, Canada \\ joey.eremondi@alumni.ubc.ca \\ Oscar H. Ibarra ${ }^{\dagger}$ \\ Department of Computer Science \\ University of California, Santa Barbara, CA 93106, USA \\ ibarra@cs.ucsb.edu \\ Ian McQuillan ${ }^{\ddagger}$ \\ Department of Computer Science, University of Saskatchewan \\ Saskatoon, SK S7N 5A9, Canada \\ mcquillan@cs.usask.ca
}

Received (Day Month Year)

Accepted (Day Month Year)

Communicated by (xxxxxxxxxx)

\begin{abstract}
A language $L$ is said to be dense if every word in the universe is an infix of some word in $L$. This notion has been generalized from the infix operation to arbitrary word operations $\varrho$ in place of the infix operation ( $\varrho$-dense, with infix-dense being the standard notion of dense). It is shown here that it is decidable, for a language $L$ accepted by a oneway nondeterministic reversal-bounded pushdown automaton, whether $L$ is infix-dense. However, it becomes undecidable for both deterministic pushdown automata (with no reversal-bound), and for nondeterministic one-counter automata. When examining suffixdensity, it is undecidable for more restricted families such as deterministic one-counter automata that make three reversals on the counter, but it is decidable with less reversals. Other decidability results are also presented on dense languages, and contrasted with a marked version called $\varrho$-marked-density. Also, new languages are demonstrated to be outside various deterministic language families after applying different deletion operations from smaller families. Lastly, bounded-dense languages are defined and examined.
\end{abstract}

Keywords: counter machines; pushdown automata; decidability; density; deletion.

*Electronic version of an article published as [International Journal of Foundations of Computer Science, 29, 02, 2018, 233-250] [10.1142/S0129054118400051] (C) [copyright World Scientific Publishing Company] [https://www.worldscientific.com/worldscinet/ijfcs]

${ }^{\dagger}$ Supported, in part, by NSF Grant CCF-1117708.

$\ddagger$ Supported, in part, by a grant from the Natural Sciences and Engineering Research Council of Canada. 


\section{Introduction}

A language $L \subseteq \Sigma^{*}$ is dense if the set of all infixes of $L$ is equal to $\Sigma^{*}[3$. This notion is relevant to the theory of codes. Indeed, a language being dense is connected with the notions of independent sets [15, maximal independent sets, codes [14, and disjunctive languages [13,19.

Dense languages have been studied in [13, 19] and generalized from density to $\varrho$-density [14, where $\varrho$ is an arbitrary word operation used in place of the infixoperation in the definition. Some common examples are prefix-dense (coinciding with left dense in [13), suffix dense (coinciding with right dense in [13), infix dense (usual notion of density), outfix dense, embedding dense, and others from [14]. Each type connects with a generalized notion of independent sets and codes.

It has long been known that universality of a language $L$ (is $L=\Sigma^{*}$ ?) is undecidable for $L$ accepted by a one-way nondeterministic one-counter automaton whose counter makes only one reversal, i.e., in an accepting computation, after decreasing the counter, it can no longer increase again [2]. This shows immediately that with the identity operation, it is undecidable if $L$ in this family is identity-dense. In contrast, the universality problem is known to be decidable for one-way deterministic reversal-bounded multicounter languages [11, but these languages are not closed under taking suffix, infix, or outfix [5. However, to decide the property of infix-density, in this paper we can show contrasting results.

(1) Infix-density is decidable for $L$ accepted by a nondeterministic pushdown automaton where the pushdown is reversal-bounded (there is at most a fixed number of switches between increasing and decreasing the size of the pushdown).

(2) Infix-density is undecidable for $L$ accepted by a nondeterministic onecounter automaton (with no reversal-bound).

(3) Infix-density is undecidable for $L$ accepted by a deterministic pushdown automaton (with no reversal-bound).

Thus, it is surprisingly possible to decide if the set of all infixes of a nondeterministic reversal-bounded pushdown automaton gives universality, when it is undecidable with the identity operator for much smaller families.

Furthermore, if the question is altered to change the type of density from infixdensity to either suffix-density or prefix-density, then it is undecidable even for nondeterministic one-counter automata that make one counter reversal (coinciding with the result for identity-density). Suffix-density is decidable however for deterministic one-counter automata that makes one counter reversal, but is undecidable when there is either two more reversals, or two counters that both make one reversal. Thus suffix-density is often impossible to decide when infix-density is decidable. Prefix density is decidable for all deterministic reversal-bounded multicounter languages.

Contrasts are made between deciding if applying an operation $\varrho$ to a language 
gives $\Sigma^{*}$ and deciding if $\$ \Sigma^{*} \$$ (with $\$ \notin \Sigma$ ) is a subset of $\varrho$ applied to $L \subseteq(\Sigma \cup$ $\{\$\})^{*}$. If this condition, $\$ \Sigma^{*} \$ \subseteq \varrho(L)$, is true, the language is said to be $\varrho$-markeddense. In contrast to infix-density, infix-marked-density is undecidable with only one-way deterministic one-counter 3-reversal-bounded languages, and for the outfix operation with many families as well. Results are summarized in Table 1

In addition, new languages $L$ are established that can be accepted by a number of automata classes (deterministic one-counter machines that are 3-reversalbounded, deterministic 2-counter machines that are 1-reversal-bounded, nondeterministic one-counter one-reversal-bounded machines), but taking any of the set of infixes, suffixes, or outfixes of $L$ produces languages that cannot be accepted by deterministic machines with an unrestricted pushdown and a fixed number of reversal-bounded counters. Hence, these deletion operations can create some very complex languages. It has been previously shown in [5] though, that the set of all infixes or suffixes of all deterministic one-counter one-reversal-bounded languages only produce deterministic reversal-bounded multicounter languages. Finally, the notion of $\varrho$-bounded-dense languages is defined and examined.

\section{Definitions}

In this section, some preliminary definitions are provided.

The set of non-negative integers is represented by $\mathbb{N}_{0}$. For $c \in \mathbb{N}_{0}$, let $\pi(c)$ be 0 if $c=0$, and 1 otherwise.

We use standard notations for formal languages, referring the reader to [8]. The empty word is denoted by $\lambda$. We use $\Sigma$ and $\Gamma$ to represent finite alphabets, with $\Sigma^{*}$ as the set of all words over $\Sigma$ and $\Sigma^{+}=\Sigma^{*}-\{\lambda\}$. For a word $w \in \Sigma^{*}$, if $w=a_{1} \cdots a_{n}$ where $a_{i} \in \Sigma, 1 \leq i \leq n$, the length of $w$ is denoted by $|w|=n$, and the reversal of $w$ is denoted by $w^{R}=a_{n} \cdots a_{1}$. Given a language $L \subseteq \Sigma^{*}$, the complement of $L$ over $\Sigma^{*}, \Sigma^{*}-L$ is denoted by $\bar{L}$.

The definitions of deterministic and nondeterministic finite automata, deterministic and nondeterministic pushdown automata, deterministic Turing Machines, and instantaneous descriptions will be used from 8 .

Notation for variations of word operations which we will use throughout the paper are presented next.

Definition 1. For a language $L \subseteq \Sigma^{*}$, the prefix, suffix, infix, and outfix operations, respectively, are defined as follows:

$$
\begin{array}{ll}
\operatorname{pref}(L)=\left\{w \mid w x \in L, x \in \Sigma^{*}\right\}, & \operatorname{suff}(L)=\left\{w \mid x w \in L, x \in \Sigma^{*}\right\}, \\
\inf (L)=\left\{w \mid x w y \in L, x, y \in \Sigma^{*}\right\}, & \operatorname{outf}(L)=\left\{x y \mid x w y \in L, w \in \Sigma^{*}\right\} .
\end{array}
$$

Different types of density are now given.

Definition 2. Let $\Sigma$ be an alphabet, and $\varrho$ an operation from $\Sigma^{*}$ to $\Sigma^{*}$. Then $L \subseteq \Sigma^{*}$ is $\varrho$-dense if $\varrho(L)=\Sigma^{*}$. 
The reader is referred to [11] and 2] for a comprehensive introduction to counter machines. A nondeterministic multicounter machine is an automaton which, in addition to having a finite set of states, has a fixed number of counters. At any point, the counters may be incremented, decremented, or queried for equality to zero. For our purposes, it will accept a word by final state.

Formally, a one-way $k$-counter machine is a tuple $M=\left(k, Q, \Sigma, \triangleleft, \delta, q_{0}, F\right)$, where $Q, \Sigma, \triangleleft, q_{0}, F$ are respectively the set of states, input alphabet, right input end-marker, initial state (in $Q$ ) and accepting states (a subset of $Q$ ). The transition function $\delta$ (defined as in [4) is a relation from $Q \times(\Sigma \cup\{\triangleleft\}) \times\{0,1\}^{k}$ to $Q \times\{\mathrm{S}, \mathrm{R}\} \times\{-1,0,+1\}^{k}$, such that if $\delta\left(q, a, c_{1}, \ldots, c_{k}\right)$ contains $\left(p, d, d_{1}, \ldots, d_{k}\right)$ and $c_{i}=0$ for some $i$, then $d_{i} \geq 0$ (to prevent negative values in any counter). The symbols $\mathrm{S}$ and $\mathrm{R}$ indicate the direction that the input tape head moves, either stay or right. Further, $M$ is deterministic if $\delta$ is a partial function. A configuration of $M$ is a $k+2$-tuple $\left(q, w \triangleleft, c_{1}, \ldots, c_{k}\right)$ representing that $M$ is in state $q$, with $w \in \Sigma^{*}$ still to read as input, and $c_{1}, \ldots, c_{k} \in \mathbb{N}_{0}$ are the contents of the $k$ counters. The derivation relation $\vdash_{M}$ is defined between configurations, where $\left(q, a w, c_{1}, \ldots, c_{k}\right) \vdash_{M}\left(p, w^{\prime}, c_{1}+d_{1}, \ldots, c_{k}+d_{k}\right)$, if $\left(p, d, d_{1}, \ldots, d_{k}\right) \in$ $\delta\left(q, a, \pi\left(c_{1}\right), \ldots, \pi\left(c_{k}\right)\right)$ where $d \in\{\mathrm{S}, \mathrm{R}\}$ and $w^{\prime}=a w$ if $d=\mathrm{S}$, and $w^{\prime}=w$ if $d=\mathrm{R}$. Let $\vdash_{M}^{*}$ be the reflexive, transitive closure of $\vdash_{M}$. A word $w \in \Sigma^{*}$ is accepted by $M$ if $\left(q_{0}, w \triangleleft, 0, \ldots, 0\right) \vdash_{M}^{*}\left(q, \triangleleft, c_{1}, \ldots, c_{k}\right)$, for some $q \in F$, and $c_{1}, \ldots, c_{k} \in \mathbb{N}_{0}$. The language accepted by $M$, denoted by $L(M)$, is the set of all words accepted by $M$. Furthermore, $M$ is $l$-reversal-bounded if it operates in such a way that in every accepting computation, the count on each counter alternates between increasing and decreasing at most $l$ times.

We will use the following notations for families of languages (and classes of one-way machines):

(1) $\operatorname{NCM}(k, l)$ for nondeterministic $l$-reversal-bounded $k$-counter languages,

(2) $\mathrm{NCM}=\bigcup_{k, l \geq 0} \operatorname{NCM}(k, l)$,

(3) NCA for nondeterministic 1-counter languages (no reversal bound),

(4) NPDA for nondeterministic pushdown languages,

(5) $\operatorname{NPDA}(l)$ for nondeterministic $l$-reversal-bounded pushdown languages,

(6) NPCM for languages accepted by nondeterministic machines with one unrestricted pushdown and a fixed number of reversal-bounded counters.

For each of the above, replacing $\mathrm{N}$ with $\mathrm{D}$ gives the deterministic variant.

It is easy to show that a counter that makes $l \geq 1$ reversals can be simulated by $\left\lceil\frac{l+1}{2}\right\rceil 1$-reversal-bounded counters 11 . So, e.g., for each $l \geq 1, \operatorname{DCM}(1, l) \subseteq$ $\operatorname{DCM}\left(\left\lceil\frac{l+1}{2}\right\rceil, 1\right)$ and thus $\operatorname{DCM}(1,3) \subseteq \operatorname{DCM}(2,1)$. Thus the undecidability results for machines with $k l$-reversal-bounded counters also carry over to machines with $k\left\lceil\frac{l+1}{2}\right\rceil 1$-reversal-bounded counters (e.g., $\operatorname{DCM}(1,3)$ to $\operatorname{DCM}(2,1)$ ).

We give some examples below to illustrate the workings of the reversal-bounded counter machines. 
Example 1. Let $L=\left\{a^{i} b^{j} a^{i} b^{j} \mid i, j \geq 1\right\}$. This language (which is not contextfree) can be accepted by a $\operatorname{DCM}(2,1)$ which, when given $a^{i} b^{j} a^{k} b^{l}$, reads the first segment $a^{i} b^{j}$ and stores $i$ and $j$ in counters $C_{1}$ and $C_{2}$, respectively. Then, it reads the next segment $a^{k} b^{l}$ and verifies that $i=k$ and $j=l$, by decrementing $C_{1}$ (resp., $C_{2}$ ) when reading $a^{k}$ (resp., $b^{l}$ ).

Example 2. The Post Correspondence Problem (PCP) [17] is the problem of deciding, given two $n$-tuples of strings $(X, Y)$, where $X=\left(x_{1}, \ldots, x_{n}\right)$ and $Y=$ $\left(y_{1}, \ldots, y_{n}\right)$ with each $x_{i}, y_{i} \in \Sigma^{+}, 1 \leq i \leq n$, whether it has a solution, i.e., whether there exists $i_{1}, \ldots, i_{k}, k \geq 1,1 \leq i_{l} \leq n$, for $1 \leq l \leq k$ such that $x_{i_{1}} \cdots x_{i_{k}}=y_{i_{1}} \cdots y_{i_{k}}$. It is known that PCP is undecidable for $|\Sigma| \geq 2$ [17].

Now consider the following variation of PCP, called Permuted PCP: Given X and $Y$ as above, does there exist $k \geq 1, I=\left(i_{1}, \ldots, i_{k}\right)$, and $J=\left(j_{1}, \ldots, j_{k}\right)$, $1 \leq i_{l} \leq n, 1 \leq j_{l} \leq n$, for $1 \leq l \leq k, I$ is a permutation of $J$, such that $x_{i_{1}} \cdots x_{i_{k}}=$ $y_{j_{1}} \cdots y_{j_{k}}$. It was shown in [9] that Permuted PCP is decidable using a restricted model of a multihead pushdown automaton whose emptiness problem is decidable. Below, we use the technique in [9] to show this result using $\mathrm{NCM} s$.

Given $(X, Y)$, let $L(X, Y)=\left\{w \mid\right.$ for some $k \geq 1, w=x_{i_{1}} \cdots x_{i_{k}}=$ $y_{j_{1}} \cdots y_{j_{k}},\left(i_{1}, \ldots, i_{k}\right)$ is a permutation of $\left.\left(j_{1}, \ldots, j_{k}\right)\right\}$. We can construct an NCM $M$ to accept $L(X, Y)$. $M$ has the tuples $X=\left(x_{1}, \ldots, x_{n}\right)$ and $Y=\left(y_{1}, \ldots, y_{n}\right)$ in its finite-state control and has $2 n$ 1-reversal-bounded counters $C_{1}, \ldots, C_{n}, D_{1}, \ldots, D_{n}$. $M$ operates as follows, given input $w$. It reads the input $w$ and, in parallel, nondeterministically guesses two decompositions of $w: w=x_{i_{1}} \cdots x_{i_{r}}$ and $w=y_{j_{1}} \cdots y_{j_{s}}$ while incrementing counter $C_{p}$ every time it guesses and verifies that $x_{i_{t}}=x_{p}$ $(t=1, \ldots, r)$ and incrementing $D_{p}$ every time it guesses and verifies that $y_{j_{t}}=y_{p}$ $(t=1, \ldots, s)$. When $M$ reaches the end of the input, it decrements the counters and accepts if and only if $C_{i}=D_{i}$ for $1 \leq i \leq n$. Since the emptiness problem for NCM is decidable [11], it follows that Permuted PCP is decidable.

Example 3. Let $(X, Y)$ be as above, and let $L^{\prime}(X, Y)=\{w \mid$ for some $k \geq 1, w=$ $x_{i_{1}} \cdots x_{i_{k}}, w^{R}=y_{j_{1}} \cdots y_{j_{k}},\left(i_{1}, \ldots, i_{k}\right)$ is a permutation of $\left.\left(j_{1}, \ldots, j_{k}\right)\right\}$. We can construct an NPCM $M^{\prime}$ with 2 n 1-reversal-bounded counters and whose stack makes only one reversal to accept $L^{\prime}(X, Y)$. The idea is for $M^{\prime}$ to guess the decomposition of $w, w=x_{i_{1}} \cdots x_{i_{r}}$ while storing the number of $x_{p}$ 's there are in string $w$ in counter $C_{p}$ and copying $w$ in the stack. When $M$ reaches the end of the input, it pops the stack and guesses the decmposition $w^{R}, w^{R}=y_{j_{1}} \cdots y_{j_{s}}$ and storing the number of $y_{p}$ 's there are in string $w^{R}$ in counter $D_{p}$. Finally, $M^{\prime}$ checks that $C_{i}=D_{i}$ for $1 \leq i \leq n$.

Example 4. Let $M$ be the NCM accepting the language $L(X, Y)$ in Example Q We can attach a pushdown stack to $M$ and obtain a NPCM $M^{\prime}$. Clearly, such a machine can accept a language $L(X, Y) \cap L$, where $L$ is a context-free language. Since the emptiness problem for NPCM is decidable [11], it follows that it is decidable, given $(X, Y)$ and an NPDA $M^{\prime \prime}$, whether $L(X, Y) \cap L\left(M^{\prime \prime}\right)=\emptyset$. 


\section{Deciding Types of Density}

In addition to examining decidability of $\varrho$-density, a variant is defined called $\varrho$ marked-density that differs from $\varrho$-density only by an end-marker.

Definition 3. Let $\Sigma$ be an alphabet, $\$ \notin \Sigma, L \subseteq(\Sigma \cup\{\$\})^{*}$, and $\varrho$ be an operation from $(\Sigma \cup\{\$\})^{*}$ to itself. Then $L$ is $\varrho$-marked-dense if $\$ \Sigma^{*} \$ \subseteq \varrho(L)$.

It is only the marker $\$$ that differs from the usual $\varrho$-dense (i.e., $\Sigma^{*} \subseteq \varrho(L)$ if and only if $\Sigma^{*}=\varrho(L)$ for $\left.L \subseteq \Sigma^{*}\right)$. Yet we will see differences, as there are cases when the marked version is undecidable when the unmarked version is decidable.

First, deciding if languages are prefix-dense will be examined. It was recently shown in [5] that DCM languages are closed under prefix. The following is a result in that paper.

Proposition 1. For $L \in \mathrm{DCM}$, $\operatorname{pref}(L) \in \mathrm{DCM}$.

A main result in that paper was in fact far more general, showing that DCM is closed (with an effective construction) under right quotient with NPCM languages. Combining this with the known decidability of the inclusion problem for DCM [11, the following two corollaries are obtained, by testing if $\Sigma^{*} \subseteq \operatorname{pref}(L)$ :

Corollary 1. For $L_{1}, L_{2} \in \mathrm{DCM}$, it is decidable whether $\operatorname{pref}\left(L_{1}\right) \subseteq L_{2}$ and whether $L_{1} \subseteq \operatorname{pref}\left(L_{2}\right)$.

Corollary 2. It is decidable whether a given DCM language is prefix-dense, and prefix-marked-dense.

This result is essentially the same for DPDA, since it was shown that DPDA is closed under prefix [7]. Then, for prefix-density, it suffices to determine if $\Sigma^{*}$ is equal to the prefix closure. And for prefix-marked-density, it suffices to determine if $\$ \Sigma^{*} \$$ is equal to the prefix closure intersected with the regular language $\$ \Sigma^{*} \$$ (DPDA is closed under intersection with regular languages [7]). And in both cases, the equality problem is decidable for DPDA [18].

Proposition 2. It is decidable whether a given DPDA language is prefix-dense, and prefix-marked-dense.

Next, it is shown in [5] that the set of suffixes and infixes of a $\operatorname{DCM}(1,1)$ language is always in DCM (by sometimes increasing the number of counters). From this, the following is obtained:

Proposition 3. For $L_{1}, L_{2} \in \operatorname{DCM}(1,1)$, it is decidable whether $\inf \left(L_{1}\right) \subseteq L_{2}$ and whether $L_{1} \subseteq \inf \left(L_{2}\right)$. It is also decidable whether $\operatorname{suff}\left(L_{1}\right) \subseteq L_{2}$ and whether $L_{1} \subseteq \operatorname{suff}\left(L_{2}\right)$.

Corollary 3. It is decidable whether a $\operatorname{DCM}(1,1)$ language is infix-dense, suffixdense, infix-marked-dense and suffix-marked-dense. 
This result will be improved shortly using a more general machine class for infixdensity, but not for suffix-density, suffix-marked-density, or infix-marked-density.

Most undecidability proofs in this section use the halting problem for Turing machines. Let $U \subseteq\{a\}^{*}$ be a unary recursively enumerable language that is not recursive, i.e., not decidable (such a $U$ exists [16]), and let $Z$ be a deterministic Turing machine accepting $U$. Assume that $Z$ accepts if and only if $Z$ halts.

Let $Q$ and $\Gamma$ be the state set and worktape alphabet of $Z$, and $q_{0} \in Q$ be the initial state of $Z$. Note that $a$ is in $\Gamma$. Let $\Sigma=Q \cup \Gamma \cup\{\#\}$. Assume without loss of generality that if $Z$ halts, it does so in a unique final state $q_{f} \neq q_{0}$, and a unique configuration, and that the initial state $q_{0}$ is never re-entered after the initial configuration, and that the length of every halting computation is even.

The halting computation of $Z$ on the input $a^{d}$ (if it accepts) can be represented by the string $x_{d}=I D_{1} \# I D_{2}^{R} \# \cdots \# I D_{k-1} \# I D_{k}^{R}$ for some $k \geq 2$, where $I D_{1}=q_{0} a^{d}$ and $I D_{k}$ are the initial and unique halting configurations of $Z$, and $\left(I D_{1}, I D_{2}, \cdots, I D_{k}\right)$ is a valid sequence of instantaneous descriptions (IDs, defined in [8) of $Z$ on input $a^{d}$, i.e., configuration $I D_{i+1}$ is a valid successor of $I D_{i}$, and $k$ is even.

Let $d \geq 0$. Let $T$ be all strings $w$ of the form $I D_{1} \# I D_{2}^{R} \# \cdots \# I D_{k-1} \# I D_{k}^{R}$, where $k \geq 2, I D_{1}=q_{0} a^{d}$, and $I D_{k}$ is the halting configuration of $Z$, and $I D_{i}$ is any ID of the Turing machine, $1<i<k$. Then $T$ is a regular language, and thus a DFA $M_{T}$ can be built accepting $T$, and also one can be built accepting $\bar{T}$. Let $L_{n a}$ be all strings $w \in T$ of the form $I D_{1} \# I D_{2}^{R} \# \cdots \# I D_{k-1} \# I D_{k}^{R}$, where there is an $i$ such that $I D_{i+1}$ is not a valid successor of $I D_{i}$. Indeed, if $I D_{i+1}$ is not a valid successor of $I D_{i}$, then this is detectable by scanning the state of $I D_{i}$, the letter after the state (symbol under the read/write head), and from these, the transition of $Z$ applied to get the valid successor of $I D_{i}$ can be calculated, as with whether the $I D$ representing the valid successor to $I D_{i}$ should be shorter or longer by one symbol. Then, there is some position $j$ of $I D_{i}$ such that examining positions $j-2, j-1, j, j+1, j+2$ of $I D_{i}$ and $I D_{i+1}$, and the state of $I D_{i}$ and $I D_{i+1}$ is enough to imply that $I D_{i+1}$ is not a valid successor. Hence, let $L_{n a}(p)$ be the set of words $w \in T$ of the form $w=I D_{1} \# I D_{2}^{R} \# \cdots \# I D_{k-1} \# I D_{k}^{R}$, where the $p$ th character of $w$ is within the string $I D_{i}$ for some $i$ at position $j$ of $I D_{i}$ and examining characters $j-2, j-1, j, j+1, j+2$ of $I D_{i}$ and $I D_{i+1}$ (if they exist), plus the states of both, and the letter after the state, implies that $I D_{i+1}$ is not a valid successor of $I D_{i}$. Thus, $\bigcup_{p \geq 1} L_{n a}(p)=L_{n a}$.

Let $L_{d}=L_{n a} \cup \bar{T}$. Two lemmas are required for undecidability results.

Lemma 1. $L_{d}=\Sigma^{*}$ if and only if $T \subseteq L_{n a}$ if and only if $Z$ does not halt on $a^{d}$.

Proof. If $L_{d}=\Sigma^{*}$, then $T \subseteq L_{n a}$, and if $T \subseteq L_{n a}$ then $T \cup \bar{T}=\Sigma^{*} \subseteq L_{d}$. Thus the first two are equivalent.

Assume $L_{d}=\Sigma^{*}$. Thus, every sequence of IDs in $T$ is in $L_{n a}$, thus there is no sequence of IDs that halts on $a^{d}$. 
Assume that $Z$ does not halt on $a^{d}$. Let $w \in \Sigma^{*}$. If $w \notin T$, then $w \in L_{d}$. If $w \in T$, then $w$ does not represent an accepting computation, thus, $w \in L_{d}$.

Let $\%$ be a new symbol not in $\Sigma$, and let $\Sigma_{\%}=\Sigma \cup\{\%\}$.

Lemma 2. $\bigcup_{p>1} \%^{p} L_{n a}(p)$ and $\bigcup_{p>1} \%^{p} \$ L_{n a}(p) \$$ are both in $\operatorname{DCM}(1,3)$ and $\operatorname{DCM}(2,1)$. Furthermore, $L_{n a}, \$ L_{n a} \$ \in \operatorname{NCM}(1,1)$.

Proof. We can construct a $\operatorname{DCM}(1,3)$ machine $M_{n a}$ to accept the strings of $\bigcup_{p \geq 1} \%^{p} L_{n a}(p)$ as follows: when given $\%^{p} w$, it reads $\%^{p}$ and increments the counter by $p$. It then decrements the counter and verifies that when the counter becomes zero, the input head is within some $I D_{i}$ (or $I D_{i}^{R}$ if $i$ is even). If $i$ is odd, $M_{n a}$ then moves the input head incrementing the counter until it reaches the \# to the right of $I D_{i}$. Let $j$ be the value of the counter. $M_{n a}$ then decrements the counter while moving right on $I D_{i+1}^{R}$ and after reaching zero, verifying that $I D_{i+1}$ is not a valid successor of $I D_{i}$ (this is possible as $I D_{i+1}^{R}$ is reversed). Similarly when $i$ is even. In the same way, we can construct a $\operatorname{DCM}(1,3)$ machine to accept $\bigcup_{p \geq 1} \%^{p} \$ L_{n a}(p) \$$. Both languages are in $\operatorname{DCM}(2,1)$ as $\operatorname{DCM}(1,3) \subseteq \operatorname{DCM}(2,1)$.

For $L_{n a}$ (and $\$ L_{n a} \$$ ), it is possible to nondeterministically guess the position $p$, and then when within $I D_{i}$, verify using the counter once that $I D_{i+1}$ is not a valid successor to $I D_{i}$.

This is similar to the technique from [2] to show undecidability of universality for $\operatorname{NCM}(1,1)$.

Most of the undecidability results in this section build off of the above two lemmas, the input $a^{d}$, the languages $T, L_{n a}$, etc.

Proposition 4. Let $\Sigma$ be an alphabet.

(1) It is undecidable to determine, given $L \in \operatorname{NCM}(1,1)$, whether $L$ is $\varrho$-markeddense, for $\varrho \in\{$ suff, inf, pref $\}$.

(2) It is undecidable to determine, given $L \in \operatorname{DCM}(1,3)$, whether $L$ is $\varrho$-markeddense, for $\varrho \in\{$ suff, inf $\}$.

(3) It is undecidable to determine, given $L \in \operatorname{DCM}(2,1)$, whether $L$ is $\varrho$-markeddense, for $\varrho \in\{$ suff, inf $\}$.

Proof. For part 1, we can accept $L^{\prime}=\$ L_{n a} \$ \cup \$ \bar{T} \$ \subseteq(\Sigma \cup\{\$\})^{*}$ in $\operatorname{NCM}(1,1)$ since $\$ \bar{T} \$$ is a regular language (the complement is over $\Sigma^{*}$ ).

Then $\$ \Sigma^{*} \$ \subseteq \inf \left(L^{\prime}\right)$ (resp., $\$ \Sigma^{*} \$ \subseteq \operatorname{suff}\left(L^{\prime}\right), \$ \Sigma \$ \subseteq \operatorname{pref}\left(L^{\prime}\right)$ ) if and only if $\$ \Sigma^{*} \$=L^{\prime}$ if and only if $L_{d}=\Sigma^{*}$, which we already know is true if and only if $Z$ does not halt on $a^{d}$ by Lemma 1 , which is undecidable.

For parts 2 and 3, we instead use $L^{\prime}=\bigcup_{p>1} \%^{p} \$ L_{n a}(p) \$ \cup \$ \bar{T} \$$, the complement $\bar{T}$ is over $\Sigma_{\%}^{*}=(\Sigma \cup\{\%\})^{*}$ here, so it will also contain any word with $\%$ in it to allow for marked-density to be with $L^{\prime} \subseteq\left(\Sigma_{\%} \cup\{\$\}\right)^{*}$ where the goal is to decide 
whether $\$ \Sigma_{\%}^{*} \$ \subseteq \inf \left(L^{\prime}\right)$. Then $L^{\prime}$ is in $\operatorname{DCM}(1,3) \cap \operatorname{DCM}(2,1)$ by Lemma 2 and since $\operatorname{DCM}(k, l)$ is closed under union with regular languages, for every $k, l[11$. And $\$ \Sigma_{\%}^{*} \$ \subseteq \inf \left(L^{\prime}\right)$ if and only if $\$ \Sigma^{*} \$ \subseteq \inf \left(L^{\prime}\right)$ (since $\bar{T}$ contains all words with at least one \%) if and only if $L_{d}=\Sigma^{*}$. The proof in the case of the suffix operation is similar.

The proof for the outfix operation is similar.

Proposition 5. It is undecidable, given $L \in \mathrm{NCM}(1,1)$, whether $L$ is outf-markeddense. Similarly with $L \in \operatorname{DCM}(2,1)$, and $L \in \operatorname{DCM}(1,3)$.

Proof. For $L \in \operatorname{NCM}(1,1)$, we modify the language $L^{\prime}$ in the proof of Part 1 of Proposition 4 So $L^{\prime}=\% \$ L_{n a} \$ \cup \% \$ \bar{T} \$\left(\bar{T}\right.$ over $\left.\Sigma_{\%}^{*}\right)$. For the other classes, $L^{\prime}$ in the proofs of parts 2, 3 also work for outf.

It follows from Propositions 4 and 5 that DPDA(3) has an undecidable $\varrho$-markeddensity problem for suffix, infix, and outfix. The following shows that they are also undecidable for DPDA(1).

Proposition 6. For $\varrho \in\{$ suff, inf, outf $\}$, it is undecidable given $L \in \operatorname{DPDA}(1)$, whether $L$ is $\varrho$-marked-dense.

Proof. The problem of whether the intersection of two DPDA(1) languages is empty is undecidable 2]. Let $L_{1}, L_{2} \in \operatorname{DPDA}(1)$. Then $L_{1} \cap L_{2}=\emptyset$ if and only if $\overline{L_{1} \cap L_{2}}=$ $\Sigma^{*}$ if and only if $\overline{L_{1}} \cup \overline{L_{2}}=\Sigma^{*}$ if and only if $\$ \Sigma^{*} \$ \subseteq \$ \overline{L_{1}} \$ \cup \$ \overline{L_{2}} \$$.

Let $L^{\prime}=\% \$ \overline{L_{1}} \$ \cup \$ \overline{L_{2}} \$ \cup \$ \Sigma_{\%}^{*} \% \Sigma_{\%}^{*} \$$ (here, the complements are over $\Sigma^{*}$ ). Note that $L^{\prime} \subseteq\left(\Sigma_{\%} \cup\{\$\}\right)^{*}=(\Sigma \cup\{\%, \$\})^{*} . L^{\prime}$ is in DPDA(1) since DPDA(1) is closed under complement, the union of the first two sets is a DPDA(1) language (if \% is the first letter then simulate the first set, otherwise simulate the second), and the third one is regular and DPDA(1) is closed under union with regular sets.

Then $\$ \Sigma_{\%}^{*} \$ \subseteq \inf \left(L^{\prime}\right)$ if and only if $\$ \Sigma_{\%}^{*} \$ \subseteq \$ \overline{L_{1}} \$ \cup \$ \overline{L_{2}} \$ \cup \$ \Sigma_{\%}^{*} \% \Sigma_{\%}^{*} \$$ if and only if $\$ \Sigma^{*} \$ \subseteq \$ \overline{L_{1}} \$ \cup \$ \overline{L_{2}} \$$, which we know is undecidable. The proof is identical for suffix, as with outfix after preceding each word in $L^{\prime}$ by an additional $\%$.

Next, $\varrho$-density instead of $\varrho$-marked-density will be considered; specifically, the question of whether it is decidable to determine if a language $L$ is $\varrho$-dense $(\varrho(L)=$ $\left.\Sigma^{*}\right)$ for various operations and languages. For suffix-density, undecidability occurs for the same families as for marked-suffix-density. The proofs will again build on the Turing Machine $Z$, input $a^{d}$, and languages $L_{n a}, T$, etc.

Proposition 7. Let $L \in \operatorname{DCM}(1,3)$. It is undecidable to determine if $L$ is suffixdense. Similarly for $L \in \mathrm{DCM}(2,1)$ and $L \in \operatorname{NCM}(1,1)$.

Proof. Let $L_{1}^{\prime}=\left\{\%^{p} u x\left|u \in \Sigma \Sigma_{\%}^{*}, p=\right| u \mid+p^{\prime}, x \in L_{n a}\left(p^{\prime}\right)\right\}, L_{2}^{\prime}=\overline{\Sigma_{\%}^{*} T}$ (over $\left.\Sigma_{\%}^{*}\right)$, and $L^{\prime}=L_{1}^{\prime} \cup L_{2}^{\prime}$. Then $L_{1}^{\prime} \in \operatorname{DCM}(1,3)$ as one can build $M_{1}^{\prime} \in \operatorname{DCM}(1,3)$ 
by adding $p$ to the counter until hitting a letter that is not $\%$. Then as $M_{1}^{\prime}$ reads the remaining input in $\Sigma \Sigma_{\%}^{*}$, for every character read, it decreases the counter, and each time $M_{1}^{\prime}$ hits state $q_{0}$ (which could be the beginning of a word in $T$ ), it runs $M_{T}$ (the DFA accepting $T$ ) in parallel to check if the suffix starting at this position is in $T$. If it hits $q_{0}$ more than once, it can stop previous simulations of $M_{T}$ and start a new simulation. However, it is only required that a suffix of the input is in $T$. If the counter empties while $M_{T}$ is running in parallel, then let $u x$ be the input, where $u$ is the input before reaching $q_{0}$ in the current run of $M_{T}$, and $x$ be the input from $q_{0}$ to the end. Then $M_{1}^{\prime}$ tries to verify that $x \in L_{n a}\left(p^{\prime}\right)$, where $p=|u|+p^{\prime}$. When the counter reaches $0, M_{1}^{\prime}$ has subtracted 1 from the counter the length of $u$ plus $p-|u|=p^{\prime}$ times. Thus, $M_{1}^{\prime}$ can continue the simulation of $M_{n a}$ from Lemma 2 from when the counter reaches 0 , thereby verifying that $x \in L_{n a}\left(p^{\prime}\right)$ (and $x \in T)$. Then $L^{\prime} \in \operatorname{DCM}(1,3)$ as each $\operatorname{DCM}(k, l)$ is closed under union with regular languages [11. Then also $L^{\prime}$ must be in $\operatorname{DCM}(2,1)$.

It will be shown that $\operatorname{suff}\left(L^{\prime}\right)=\Sigma_{\%}^{*}$ if and only if $T \subseteq L_{n a}$, which is enough by Lemma 1

" $\Leftarrow$ " Assume $T \subseteq L_{n a}$. Let $w \in \Sigma_{\%}^{*}$.

Assume that there exists a (potentially not proper) suffix of $w$ in $T$. Then $w=u x, x \in T, u \in \Sigma_{\%}^{*}$. Then $x \in L_{n a}$, by assumption. Then there exists $p$ such that $\%^{p} x \in L_{n a}(p), x \in T$ and so $\%^{p^{\prime}}$ aux $\in L_{1}^{\prime}$, au $\in \Sigma \Sigma_{\%}^{*}$, where $p^{\prime}=p+|a u|$. Thus $u x=w \in \operatorname{suff}\left(L_{1}^{\prime}\right)$.

Assume that there does not exist a suffix of $w$ in $T$. Then $w \in L_{2}^{\prime}$, and $w \in$ $\operatorname{suff}\left(L^{\prime}\right)$.

" $\Rightarrow$ " Assume $\operatorname{suff}\left(L^{\prime}\right)=\Sigma_{\%}^{*}$. Let $w \in T$. Then $w \in \operatorname{suff}\left(L^{\prime}\right)$. Then there exists $\%^{p} u w \in L_{1}^{\prime}$. This implies there exists $p^{\prime}$ such that $w \in L_{n a}\left(p^{\prime}\right) \subseteq L_{n a}$.

The case for $\operatorname{NCM}(1,1)$ is similar except using $L_{1}^{\prime}=\left\{u x \mid x \in L_{n a}, u \in \Sigma^{*}\right\}$ and $L_{2}^{\prime}=\overline{\Sigma^{*} T}$, and $L^{\prime}=L_{1}^{\prime} \cup L_{2}^{\prime} \subseteq \Sigma^{*}$, as $u$ can be nondeterministically guessed without using the counter.

Corollary 4. For $L \in \operatorname{NCM}(1,1)$, the question of whether $L$ is prefix-dense is undecidable.

Proof. It is known that $\operatorname{NCM}(k, l)$ is closed under reversal for each $k, l$. Also, $\operatorname{pref}\left(L^{R}\right)=\Sigma^{*}$ if and only if $\operatorname{suff}(L)=\Sigma^{*}$.

We are able to extend the undecidability results to infix-density, but only by using one unrestricted counter and with nondeterminism.

Proposition 8. Let $L \in$ NCA. The question of whether $L$ is infix-dense is undecidable.

Proof. Let $L^{\prime}=\overline{\left(\Sigma^{*} T \Sigma^{*}\right)}\left(L_{n a} \overline{\left(\Sigma^{*} T \Sigma^{*}\right)}\right)^{*} \subseteq \Sigma^{*}$. It is clear that $L^{\prime} \in$ NCA. We will show that $T \subseteq L_{n a}$ if and only if $\inf \left(L^{\prime}\right)=\Sigma^{*}$. 
" $\Rightarrow$ " Assume $T \subseteq L_{n a}$. Let $w \in \Sigma^{*}$. If $w \in \overline{\left(\Sigma^{*} T \Sigma^{*}\right)}$, then $w \in L^{\prime} \subseteq \inf \left(L^{\prime}\right)$. Assume $w \notin \overline{\left(\Sigma^{*} T \Sigma^{*}\right)}$. Then $w \in \Sigma^{*} T \Sigma^{*}$. Then $w=u_{0} v_{1} u_{1} \cdots u_{n-1} v_{n} u_{n}$, where $n \geq 1, v_{1}, \ldots, v_{n} \in T$, and $u_{0}, \ldots, u_{n} \notin \Sigma^{*} T \Sigma^{*}$, and so $u_{0}, \ldots, u_{n} \in \overline{\left(\Sigma^{*} T \Sigma^{*}\right)}$. Also, $T \subseteq L_{n a}$, and therefore $v_{1}, \ldots, v_{n} \in L_{n a}$ and $w \in L^{\prime} \subseteq \inf \left(L^{\prime}\right)$.

" $\Leftarrow$ " Assume $\inf \left(L^{\prime}\right)=\Sigma^{*}$. Let $w \in T$. Then $w \in \inf \left(L^{\prime}\right)$. Since $w \in \inf \left(L^{\prime}\right) \cap T$, then $x=u w v \in L^{\prime}$. Then $x=u_{0} v_{1} u_{1} \cdots u_{n-1} v_{n} u_{n}$, where $n \geq 1, v_{1}, \ldots, v_{n} \in L_{n a}$, and $u_{0}, \ldots, u_{n} \in \overline{\Sigma^{*} T \Sigma^{*}}$. If $w$ is an infix of $u_{i}$, for some $i$, then $u_{i} \in \Sigma^{*} T \Sigma^{*}$, a contradiction. If $w$ overlaps with $v_{i}$ for some $i$, then it must be exactly one $v_{i}$ by the structure of $T$ (initial and final states are only used once at beginning and end of words in $T)$. Then $w \in L_{n a}$.

The same undecidability is obtained with determinism, but an unrestricted pushdown automaton is used.

Proposition 9. Let $L \in$ DPDA. The question of whether $L$ is infix-dense is undecidable.

Proof. Let $\Sigma_{1}=\Sigma \cup\{\%, e, \mathrm{c}\}$. Let

$$
\begin{aligned}
& L^{\prime}=\left\{r_{m} r_{m-1} \cdots r_{1} \dot{c} u_{0} y_{1} u_{1} \cdots y_{m} u_{m} \mid m \geq 0, u_{i} \in \overline{\Sigma_{1}^{*} T \Sigma_{1}^{*}}, 0 \leq i \leq m,\right. \\
& \left.y_{j} \in T, r_{j}=\%^{p_{j}} e^{q_{j}}, q_{j}=\left|u_{j-1}\right|, y_{j} \in L_{n a}\left(p_{j}\right) \text { for } 1 \leq j \leq m\right\} .
\end{aligned}
$$

(In the above set, the complementation is over $\Sigma_{1}^{*}$.) First, $L^{\prime}$ can be accepted by a DPDA as follows: create $M^{\prime}$ that reads $r_{m} \cdots r_{1}$ and pushes each symbol onto the pushdown, which is now (with bottom of pushdown marker $Z_{0}$ )

$$
Z_{0} \%^{p_{m}} e^{q_{m}} \ldots \%^{p_{1}} e^{q_{1}} .
$$

Then for each $\%^{p_{j}} e^{q_{j}}$ on the pushdown from 1 to $m, M^{\prime}$ reads one symbol at a time

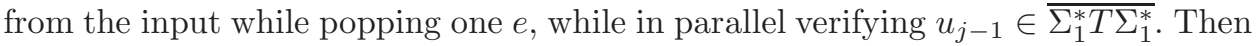
$M^{\prime}$ verifies that $y_{j} \in L_{n a}\left(p_{j}\right)$ as in Lemma 2 (by popping $\%^{p_{j}}$ one symbol at a time until zero and then pushing on the pushdown simulating the counter). Finally $M^{\prime}$ verifies $u_{m} \in \overline{\Sigma_{1}^{*} T \Sigma_{1}^{*}}$.

We claim that $\inf \left(L^{\prime}\right)=\Sigma_{1}^{*}$ if and only if $T \subseteq L_{n a}$.

Assume $T \subseteq L_{n a}$. Let $w \in \Sigma_{1}^{*}$. We will show $w \in \inf \left(L^{\prime}\right)$. Let $w=$ $u_{0} y_{1} u_{1} \cdots u_{m-1} y_{m} u_{m}$, where $m \geq 0, y_{1}, \ldots, y_{m} \in T, u_{0}, \ldots, u_{m} \in \overline{\Sigma_{1}^{*} T \Sigma_{1}^{*}}$. Then for each $y_{j}, 1 \leq j \leq m, y_{j} \in L_{n a}\left(p_{j}\right)$, for some $p_{j}$, and thus there exists $q_{j}$ such that $q_{j}=\left|u_{j-1}\right|$. Thus, $\%^{p_{m}} e^{q_{m}} \ldots \%^{p_{1}} e^{q_{1}} \dot{c} w \in L^{\prime}$, and $w \in \inf \left(L^{\prime}\right)$.

Assume $\inf \left(L^{\prime}\right)=\Sigma_{1}^{*}$. Let $w \in T$. Then there must exist $x, y$ such that $z=x w y \in$ $L^{\prime}$. Then $z=u_{0} y_{1} u_{1} \cdots u_{m-1} y_{m} u_{m}$, where $y_{1}, \ldots, y_{m} \in T, u_{0}, \ldots, u_{m} \in \overline{\Sigma_{1}^{*} T \Sigma_{1}^{*}}$. Necessarily, one of $y_{1}, \ldots, y_{m}, y_{i}$ say, must be $w$. This implies $w=y_{i} \in L_{n a}\left(p_{i}\right)$, for some $p_{i}$. Hence, $w \in L_{n a}$.

In contrast to the undecidability of marked-infix-density and suffix-density for $\operatorname{DCM}(1,3)$ and $\operatorname{NCM}(1,1)$, for infix-density on reversal-bounded nondeterministic pushdown automata, it is decidable. The main tool of the proof is the known fact 
that the language of all words over the pushdown alphabet that can appear on the pushdown in an accepting computation is a regular language [1].

Proposition 10. It is decidable, given $L$ accepted by a one-way reversal-bounded NPDA, whether $L$ is infix-dense.

Proof. Let $M=\left(Q, \Sigma, \Gamma, \delta, q_{0}, Z_{0}, F\right)$ be a pushdown automaton that accepts by final state and never pops $Z_{0}$. The pushdown is said to be empty if $Z_{0}$ is at the top of the pushdown. Also, assume $M$ makes at most $l$ switches between increasing and decreasing the size of the pushdown. Assume without loss of generality that $Q$ is partitioned into sets $Q=\bigcup_{0 \leq i \leq l} Q_{i} \cup \bigcup_{0 \leq i \leq l} \bar{Q}_{i}$, where $Q_{i}$ consists of all states defined on or after the $i$ th reversal and before the $i+1$ st on a non-empty pushdown, and $\bar{Q}_{i}$ is the same on an empty pushdown. Also, assume without loss of generality that all transitions either push one letter, keep the stack the same, or pop one letter, and there are no $\lambda$-transitions that do not change the pushdown.

Let $q \in Q_{i} \cup \bar{Q}_{i}$ for some $i$. Let $L_{q}$ be the language

$$
\left\{w \mid\left(q_{0}, u w v, Z_{0}\right) \vdash_{M}^{*}(q, w v, \alpha) \vdash_{M}^{*}(q, v, \beta) \vdash_{M}^{*}\left(q_{f}, \lambda, \gamma\right), q_{f} \in F, \alpha, \beta, \gamma \in \Gamma^{*}\right\} .
$$

It will be shown that $L^{q}$ is a regular language, for all $q \in Q$. Let $h_{\Sigma}$ be the homomorphism from $(\Sigma \cup \Gamma)^{*}$ to $\Sigma$ that erases all letters of $\Gamma$ that fixes all letters of $\Sigma$, and let $h_{\Gamma}$ be the homomorphism that erases all letters of $\Sigma$ and fixes all letters of $\Gamma$.

Consider the languages

$$
\operatorname{Acc}(q)=\left\{\alpha \in \Gamma^{*} \mid\left(q_{0}, u, Z_{0}\right) \vdash_{M}^{*}(q, \lambda, \alpha), u \in \Sigma^{*}\right\} .
$$

and

$$
\operatorname{co}-A c c(q)=\left\{\beta \in \Gamma^{*} \mid(q, v, \beta) \vdash_{M}^{*}\left(q_{f}, \lambda, \gamma\right), v \in \Sigma^{*}, q_{f} \in F\right\} .
$$

It is shown in [1] that both of these languages are in fact regular languages. Moreover, the proofs contain effective constructions.

Then consider $L^{q}, q \in \bar{Q}_{i}$. If either $\operatorname{Acc}(q)$ or $\operatorname{co}-\operatorname{Acc}(q)$ are empty, then so is $L^{q}$. If both are non-empty, then $L_{q}$ can be accepted by simulating $M$ with an NFA which can be done since all transitions are on $Z_{0}$.

Consider $L^{q}, q \in Q_{i}, i$ even. Thus, there are no decreasing transitions or transitions on an empty pushdown defined between states $q$ and $q$. Create an interim NFA $M^{\prime}$ accepting an interim language $L_{1}^{q} \subseteq \Gamma^{+}(\Sigma \cup \Gamma)^{*}$, where $M^{\prime}$ does the following in parallel:

- Nondeterministically guesses a partition of the input into $\alpha y$, where $\alpha \in$ $\Gamma^{+}, y \in(\Sigma \cup \Gamma)^{*}$, verifies that $\alpha \in A c c(q)$, and $M^{\prime}$ also remembers the last letter of $\Gamma$ in $\alpha$, and as it reads $y$, continues to remember the previous symbol from $\Gamma$ encountered. Then it reads the remaining input $y$ and starting in state $q$, simulates $M$ as follows: if the next letters are $a \in \Sigma$ followed by $d \in \Gamma$, then $M^{\prime}$ simulates (just by reading these letters $a d$ and switching 
states appropriately) a transition that reads $a$ that is defined on the remembered pushdown letter while pushing $d$ on the pushdown. Otherwise, if the next letter is $a \in \Sigma$ and a letter from $\Gamma$ does not follow, then $M^{\prime}$ simulates a transition that reads $a$ and does not push on the remembered pushdown letter. If instead the next letter is $d \in \Gamma$, then $M^{\prime}$ simulates a pushing of $d$ on $\lambda$ input on the remembered pushdown letter. At the end of the input, the simulated machine $M$ must be in state $q$.

- Also, $M^{\prime}$ reads the input, and if $h_{\Gamma}(\alpha y)=\beta$ (this is $\alpha$ plus the word from $\Gamma$ shuffled into $y$ ), then $M^{\prime}$ verifies that $\beta \in \operatorname{co}-A c c(q)$.

Claim 1. $h_{\Sigma}\left(L_{1}^{q}\right)=L^{q}$.

Proof. " $\subseteq$ " Let $s \in h_{\Sigma}\left(L_{1}^{q}\right)$. Thus, there exists $t \in(\Sigma \cup \Gamma)^{*}$ such that $h_{\Sigma}(t)=s$ and $t \in L_{1}^{q}$. Then $t=\alpha y, \alpha \in \Gamma^{+}, y \in(\Sigma \cup \Gamma)^{*}$, where $M^{\prime}$ verifies $\alpha \in A c c(q)$. Then $\left(q_{0}, u, Z_{0}\right) \vdash_{M}^{*}(q, \lambda, \alpha)$ for some $u \in \Sigma^{*}$. Then, on each letter of $y, M^{\prime}$ simulates $M$ on the last letter read from $\Gamma$, reading each letter from $\Sigma$ and pushing each letter from $\Gamma$ read starting and finishing in state $q$. Thus,

$$
\left(q, h_{\Sigma}(y), \alpha\right) \vdash_{M}^{*}\left(q, \lambda, \alpha h_{\Gamma}(y)\right)=\left(q, \lambda, h_{\Gamma}(\alpha y)\right) .
$$

Then since $M^{\prime}$ verified $h_{\Gamma}(\alpha y) \in \operatorname{co-Acc}(q)$, this implies $\left(q, v, h_{\Gamma}(\alpha y)\right) \vdash_{M}^{*}$ $\left(q_{f}, \lambda, \gamma\right), q_{f} \in F, v \in \Sigma^{*}$. Hence $h_{\Sigma}(y) \in L^{q}$ and $s=h_{\Sigma}(y)$.

" $\supseteq$ " Let $s \in L^{q}$. Thus,

$$
\left(q_{0}, u s v, Z_{0}\right) \vdash_{M}^{*}(q, s v, \alpha) \vdash_{M}^{*}(q, v, \beta) \vdash_{M}^{*}\left(q_{f}, \lambda, \gamma\right),
$$

$q_{f} \in F$. Then $\beta=\alpha \mu$, for some $\mu \in \Gamma^{*}$. Let

$$
\left(p_{0}=q, s_{0}=s v, \alpha_{0}=\alpha\right) \vdash_{M}\left(p_{1}, s_{1}, \alpha_{1}\right) \vdash_{M} \cdots \vdash_{M}\left(p_{n}=q, s_{n}=v, \alpha_{n}=\beta\right),
$$

be the derivation above between states $q$ and $q$ via transitions $t_{1}, \ldots, t_{n}$ respectively. Let $y$ be obtained by examining each $t_{i}$ in order, from 1 to $n$, and concatenating $a \in \Sigma$ if $t_{i}$ consumes $a$ ( $\lambda$ otherwise), $d \in \Gamma$ if $t_{i}$ pushes $d$ ( $\lambda$ otherwise). Then $\mu=h_{\Gamma}(y)$. We will show $\alpha y \in L\left(M^{\prime}\right)$. Indeed, $\alpha \in A c c(q)$, and $M^{\prime}$ simulates $M$ when reading $y$ ending in state $q$, and verifies that $\beta=\alpha \mu \in \operatorname{co}-\operatorname{Acc}(q)$. Thus, $s \in h_{\Sigma}\left(L_{1}^{q}\right)$.

Similarly, if $L^{q}, q \in Q_{i}, i$ odd, then there are no pushing transitions or transitions on empty pushdown between states $q$ and $q$. Then create an interim NFA $M^{\prime}$ accepting an interim language $L_{2}^{q} \subseteq(\Sigma \cup \Gamma)^{*} \Gamma^{+}$, where $M^{\prime}$ does the following in parallel:

- Nondeterministically guesses a partition of the input into $y \alpha$, where $\alpha \in \Gamma^{+}$, and simulates $M$ from state $q$. While reading a letter $a \in \Sigma$ followed by a letter from $\Gamma, M^{\prime}$ simulates a transition of $M$ that reads input letter $a \in \Sigma$ and pops the letter from $\Gamma$. Otherwise, if reading $a \in \Sigma$ not followed by a letter from $\Gamma$, then $M^{\prime}$ guesses the next letter $d \in \Gamma$ that will appear in 
$y \alpha$, simulate a transition reading $a$ with $d$ on top of the pushdown that does not change the pushdown, and eventually verify that $d$ is the next letter of $\Gamma$. If reading $d \in \Gamma$ only, then $M^{\prime}$ simulates a $\lambda$-transition that pops $d \in \Gamma$. Then, when reaching the end of $y$, it verifies that the simulated machine is in state $q$, and verifies that the remaining input $\alpha \in \operatorname{co}-\operatorname{Acc}(q)^{R}$ (as $c o-A c c(q)$ is a regular language, so is its reversal).

- Also, $M^{\prime}$ reads the input and if $h_{\Gamma}(y \alpha)=\beta$, then $M^{\prime}$ verifies that $\beta \in$ $\operatorname{Acc}(q)^{R}$.

Claim 2. $h_{\Sigma}\left(L_{2}^{q}\right)=L^{q}$.

Proof. "ᄃ" Let $s \in h_{\Sigma}\left(L_{2}^{q}\right)$. Thus, there exists $t \in(\Sigma \cup \Gamma)^{*}$ such that $h_{\Sigma}(t)=s$ and $t \in L_{2}^{q}$. Then $t=y \alpha, \alpha \in \Gamma^{+}, y \in(\Sigma \cup \Gamma)^{*}$. On each letter of $y, M^{\prime}$ simulates $M$ from state $q$ on a top-of-pushdown letter that is nondeterministically guessed, then later verified when hitting the next letter of $\Gamma$ (the topmost symbol of the pushdown), on input letters from $\Sigma$ that are read, and reading letters from $\Gamma$ that are popped, ending in $q$. Further, $M^{\prime}$ verifies that $\alpha \in \operatorname{co}-A c c(q)^{R}, h_{\Gamma}(y \alpha)=\beta \in A c c(q)^{R}$. Indeed, letters are read in $M^{\prime}$ in the same order that they are popped, which is reversed from the languages $A c c(q)$ and $c o-A c c(q)$, which are the words that appear on the pushdowns from the bottom towards the top. Hence, there exists $u, v \in \Sigma^{*}$ such that

$$
\left(q_{0}, u s v, Z_{0}\right) \vdash_{M}^{*}\left(q, s v, \beta^{R}\right) \vdash_{M}^{*}\left(q, v, \alpha^{R}\right) \vdash_{M}^{*}\left(q_{f}, \lambda, \gamma\right),
$$

$q_{f} \in F$ since $\beta^{R}=h_{\Gamma}\left(\alpha^{R} y^{R}\right)$. Hence, $s \in L^{q}$.

" $\supseteq$ " Let $s \in L^{q}$. Thus,

$$
\left(q_{0}, u s v, Z_{0}\right) \vdash_{M}^{*}\left(q, s v, \beta^{R}\right) \vdash_{M}^{*}\left(q, v, \alpha^{R}\right) \vdash_{M}^{*}\left(q_{f}, \lambda, \gamma\right),
$$

$q_{f} \in F$. Therefore, $\beta^{R}=\alpha^{R} \mu^{R}$ for some $\mu \in \Gamma^{*}$. Let

$$
\left(p_{0}=q, s_{0}=s v, \alpha_{0}=\beta^{R}\right) \vdash_{M}\left(p_{1}, s_{1}, \alpha_{1}\right) \vdash_{M} \cdots \vdash_{M}\left(p_{n}=q, s_{n}=v, \alpha_{n}=\alpha^{R}\right)
$$

be the derivation between the two configurations with $q$ above, via transitions $t_{1}, \ldots, t_{n}$ respectively. Let $y$ be obtained by examining each $t_{i}$ in order from 1 to $n$ and concatenating $a \in \Sigma$ if $t_{i}$ consumes $a$ ( $\lambda$ otherwise), and $d \in \Gamma$ if $t_{i}$ pops $d$ ( $\lambda$ otherwise). Then $\mu^{R}=h_{\Gamma}(y)$. We will show $y \alpha^{R} \in L\left(M^{\prime}\right)$. Indeed, $M^{\prime}$ verifies $\beta=\mu \alpha \in \operatorname{Acc}(q)^{R}$ and in parallel, $M^{\prime}$ simulates $M$ from $q$, reading $y$ ending in state $q$ and verifies that $\alpha \in \operatorname{Acc}(q)^{R}$. Further, $s=h_{\Sigma}(y \alpha)$ and hence, $s \in h_{\Sigma}\left(L_{2}^{q}\right)$.

Hence, $L^{q}$ is regular for all $q \in Q$ since regular languages are closed under homomorphism. Let $L^{\prime}=\bigcup_{q \in Q} L^{q}$, which is also regular.

To conclude, it will be shown that $\inf (L)=\Sigma^{*}$ if and only if $\inf \left(L^{\prime}\right)=\Sigma^{*}$.

Assume that $\inf (L)=\Sigma^{*}$. Let $w \in \Sigma^{*}$. Then $w \in \inf (L)$. Consider $w^{\prime}=w^{|Q|+1}$. Then $w^{\prime} \in \inf (L)$, and by the pigeonhole principal, an entire copy of $w$ has to be read between some state $q$ and itself. Then $w \in \inf \left(L^{q}\right)$. 
The converse is trivial since $\inf \left(L^{\prime}\right) \subseteq \inf (L)$.

Next, we briefly examine the reverse containments when testing if $\Sigma^{*} \subseteq \varrho(L)$ and $\$ \Sigma^{*} \$ \subseteq \varrho(L)$ for density and marked-density. Here, it is checked whether it is decidable to test $\varrho(L) \subseteq R$ for regular languages $R$. In fact, we will show a stronger result.

Proposition 11. It is decidable, given $L_{1} \in \mathrm{NPCM}$ and $L_{2} \in \mathrm{DCM}$, whether $\varrho\left(L_{1}\right) \subseteq L_{2}$, where $\varrho \in\{$ suff, inf, pref, outf $\}$.

Proof. It is easy to show that if $L_{1}$ is accepted by an NPCM, then $\varrho\left(L_{1}\right)$ can be accepted by an NPCM. We can also construct a DCM that accepts $\overline{L_{2}}$ [1]. We can then construct an NPCM accepting $\varrho\left(L_{1}\right) \cap \overline{L_{2}}$ as NPCM is closed under intersection with NCM. The decidability of whether $\varrho(L) \subseteq L_{2}$ is equivalent to the question of whether the NPCM accepting $\varrho(L) \cap \overline{L_{2}}$ is empty, which is decidable, since the emptiness problem for NPCMs is decidable [11].

The languages used in the proofs of Lemmas 1 and 2 are used next to show that $\varrho(L)$ does not belong in the same family as $L$, in general.

Proposition 12. There is a language $L \in \operatorname{DCM}(1,3)$ (resp., $\operatorname{NCM}(1,1)$, $\operatorname{DCM}(2,1))$ such that $\varrho(L)$ is not in DPCM, where $\varrho \in\{$ suff, inf, outf $\}$.

Proof. We first give a proof for $\operatorname{DCM}(1,3)$. Consider $L^{\prime}=\bigcup_{p \geq 1} \%^{p} \$ L_{n a}(p) \$ \in$ $\operatorname{DCM}(1,3)$ by Lemma 2 For $\varrho \in\{$ suff, inf, outf $\}$, we claim that $\varrho\left(L^{\prime}\right)$ cannot be accepted by any DPCM. We know $\$ L_{n a} \$ \subseteq \varrho\left(L^{\prime}\right)$. For suppose $\varrho\left(L^{\prime}\right)$ can be accepted by a DPCM $M_{1}$. Then, since the family of languages accepted by DPCMs is closed under complementation [10, we can construct a DPCM $M_{2}$ accepting $\overline{L\left(M_{1}\right)}$. Now using $M_{2}$, an algorithm can be constructed to determine whether $\$ T \$ \nsubseteq \$ L_{n a} \$$, which we know is a subset of $\varrho\left(L^{\prime}\right)$.

(1) Consider $T$, which can be accepted by a DFA $M_{3}$.

(2) Construct a DPCM $M_{4}$ accepting $L\left(M_{2}\right) \cap L\left(M_{3}\right)$.

(3) Check if the language accepted by $M_{4}$ is empty. This is possible since the emptiness problem for NPCMs (hence also for DPCMs) is decidable [1].

By Lemma 1, $a^{d} \in L(Z)$ if and only if $\$ T \$ \not \$ L_{n a} \$$ if and only if the language accepted by $L\left(M_{4}\right)$ is not empty. It follows that $\varrho(L) \notin$ DPCM.

Similarly with $\$ L_{n a} \$$ for $\operatorname{NCM}(1,1)$ for suffix and infix, and $\% \$ L_{n a} \$$ for outfix.

We note that the proof above also shows that if $L \in \operatorname{NCM}(1,1)$, then $\operatorname{pref}(L)$ need not be in DPCM. 


\section{Bounded-Dense Languages}

Let $\varrho$ be an operation from $\Sigma^{*}$ to $\Sigma^{*}$. Then a language $L$ is $\varrho$-bounded-dense over given words $w_{1}, \ldots, w_{k}$ if $\varrho(L)=w_{1}^{*} \cdots w_{k}^{*}$. We will show below that determining bounded-denseness is decidable for NPCM languages.

The following lemma is a generalization of a similar result for NPDAs in [6]:

Lemma 3. It is decidable, given two NPCMs $M_{1}$ and $M_{2}$, one of which accepts a bounded language that is a subset of $w_{1}^{*} \cdots w_{k}^{*}$ (for given words $w_{1}, \ldots, w_{k} \in \Sigma^{+}$), whether $L\left(M_{1}\right) \subseteq L\left(M_{2}\right)$.

Proof. We consider two cases.

Case 1: Suppose $L\left(M_{2}\right) \subseteq w_{1}^{*} \cdots w_{k}^{*}$. From a bounded NPCM language, it is known that we can construct a DCM machine $M_{2}^{\prime}$ equivalent to $M_{2}$ [12. Then, we can also construct a DCM $M_{2}^{\prime \prime}$ accepting $\overline{L\left(M_{2}^{\prime}\right)}$ 4. Next, we construct an NPCM $M$ that simulates $M_{1}$ and $M_{2}^{\prime \prime}$ in parallel to accept $L\left(M_{1}\right) \cap L\left(M_{2}^{\prime \prime}\right)$. Clearly, $L\left(M_{1}\right) \subseteq L\left(M_{2}\right)$ if and only if $L(M)=\emptyset$, which is decidable since the emptiness problem for NPCMs is decidable [11].

Case 2: Suppose $L\left(M_{1}\right) \subseteq w_{1}^{*} \cdots w_{k}^{*}$. First we construct an NPCM $M_{2}^{\prime}$ that accepts $L\left(M_{2}\right) \cap w_{1}^{*} \cdots w_{k}^{*}$. Then $L\left(M_{1}\right) \subseteq L\left(M_{2}\right)$ if and only if $L\left(M_{1}\right) \subseteq L\left(M_{2}^{\prime}\right)$, which is decidable by Case 1 .

Corollary 5. It is decidable, given two NPCMs $M_{1}, M_{2}$ accepting bounded languages $L\left(M_{1}\right), L\left(M_{2}\right) \subseteq w_{1}^{*} \cdots w_{k}^{*}$, whether $L\left(M_{1}\right) \subseteq L\left(M_{2}\right)$ (resp., $L\left(M_{1}\right)=$ $\left.L\left(M_{2}\right)\right)$.

Let $\varrho \in\{$ suff, inf, pref, outf $\}$. Clearly, if $M$ is an NPCM accepting a language $L(M) \subseteq w_{1}^{*} \cdots w_{k}^{*}$, we can construct an NPCM $M^{\prime}$ such that $L\left(M^{\prime}\right)=\varrho(L(M))$, and $L\left(M^{\prime}\right)$ is bounded, but over $v_{1}^{*} \cdots v_{l}^{*}$, which are effectively constructable from $w_{1}, \ldots, w_{k}$. From Lemma 3 , by testing $w_{1}^{*} \cdots w_{k}^{*} \subseteq L\left(M^{\prime}\right)$ we have:

Proposition 13. Let $\varrho \in\{$ pref, inf, suff, outf $\}$. It is decidable, given an NPCM $M$ accepting a language $L(M) \subseteq w_{1}^{*} \cdots w_{k}^{*}$ (for given $w_{1}, \ldots, w_{k}$ ), whether $L(M)$ is @-bounded-dense.

\section{Conclusions}

This paper studies decidability problems involving testing whether a language $L$ is $\varrho$-dense and $\varrho$-marked-dense, depending on the language family of $L$. For the prefix operation, all are decidable for DCM, but undecidable for $\operatorname{NCM}(1,1)$, and thus the problem has been completely characterized in terms of restrictions on reversalbounded multicounter machines. For suffix, both density and marked-density are decidable for $\operatorname{DCM}(1,1)$, but not for $\operatorname{DCM}(1,3)$ and $\operatorname{NCM}(1,1)$, and therefore this has also been completely characterized. For infix, marked-density is decidable for 


\begin{tabular}{|l|c|c|c|c|}
\hline unmarked density & infix & suffix & prefix & outfix \\
\hline \hline DCM $(1,1)$ & $\checkmark_{C 3}$ & $\checkmark_{C 3}$ & $\checkmark_{C 2}$ & $?$ \\
\hline DCM $(1,3)$ & $\checkmark_{P 10}$ & $\times_{P 7}$ & $\checkmark_{C 2}$ & $?$ \\
\hline DCM $(2,1)$ & $?$ & $\times_{P 7}$ & $\checkmark_{C 2}$ & $?$ \\
\hline DCM & $?$ & $\times_{P 7}$ & $\checkmark_{C 2}$ & $?$ \\
\hline DPDA $(1)$ & $\checkmark_{P 10}$ & $?$ & $\checkmark_{P 2}$ & $?$ \\
\hline DPDA & $\times_{P 9}$ & $\times_{P 7}$ & $\checkmark_{P 2}$ & $?$ \\
\hline NCM $(1,1)$ & $\checkmark_{P 10}$ & $\times_{P 7}$ & $\times_{C 4}$ & $?$ \\
\hline NCM & $?$ & $\times_{P 7}$ & $\times_{C 4}$ & $?$ \\
\hline NCA & $\times_{P 8}$ & $\times_{P 7}$ & $\times_{C 4}$ & $?$ \\
\hline rev-NPDA & $\checkmark_{P 10}$ & $\times_{P 7}$ & $\times_{C 4}$ & $?$ \\
\hline NPDA & $\times_{P 8}$ & $\times_{P 7}$ & $\times_{C 4}$ & $?$ \\
\hline \hline marked density & infix & suffix & $p_{e f i x}$ & outfix \\
\hline \hline DCM $(1,1)$ & $\checkmark_{C 3}$ & $\checkmark_{C 3}$ & $\checkmark_{C 2}$ & $?$ \\
\hline DCM $(1,3)$ & $\times_{P 4}$ & $\times_{P 4}$ & $\checkmark_{C 2}$ & $\times_{P 5}$ \\
\hline DCM $(2,1)$ & $\times_{P 4}$ & $\times_{P 4}$ & $\checkmark_{C 2}$ & $\times_{P 5}$ \\
\hline DCM & $\times_{P 4}$ & $\times_{P 4}$ & $\checkmark_{C 2}$ & $\times_{P 5}$ \\
\hline DPDA $(1)$ & $\times_{P 6}$ & $\times_{P 6}$ & $\checkmark_{P 2}$ & $\times_{P 6}$ \\
\hline DPDA & $\times_{P 6}$ & $\times_{P 6}$ & $\checkmark_{P 2}$ & $\times_{P 6}$ \\
\hline NCM $(1,1)$ & $\times_{P 4}$ & $\times_{P 4}$ & $\times_{P 4}$ & $\times_{P 5}$ \\
\hline NCM & $\times_{P 4}$ & $\times_{P 4}$ & $\times_{P 4}$ & $\times_{P 5}$ \\
\hline NCA & $\times_{P 4}$ & $\times_{P 4}$ & $\times_{P 4}$ & $\times_{P 5}$ \\
\hline rev-NPDA & $\times_{P 4}$ & $\times_{P 4}$ & $\times_{P 4}$ & $\times_{P 5}$ \\
\hline NPDA & $\times_{P 4}$ & $\times_{P 4}$ & $\times_{P 4}$ & $\times_{P 5}$ \\
\hline
\end{tabular}

Table 1. Summary of results, in the top half of the table with different types of density, and in the bottom half of the table with different types of marked density. A checkmark represents decidability, a cross is undecidable, and a question mark represents an open problem. The proposition proving each result is listed as subscript (with $\mathrm{C}$ being a corollary, and $\mathrm{P}$ a proposition).

$\operatorname{DCM}(1,1)$, but not for $\operatorname{DCM}(1,3)$ and $\operatorname{NCM}(1,1)$. For infix-density however, it is decidable for nondeterministic reversal-bounded pushdown automata, but undecidable for deterministic pushdown automata and nondeterministic one-counter automata. It remains open for DCM and NCM when there are at least two counters, and also for deterministic one-counter automata. For outfix, marked-density is undecidable for $\operatorname{DCM}(1,3), \operatorname{DCM}(2,1)$ and $\operatorname{NCM}(1,1)$ but is open for $\operatorname{DCM}(1,1)$. All variants are open for outfix-density.

In Section 4, results on bounded-dense languages are presented where the words $w_{1}, \ldots, w_{k}$ are given. In particular, for each of prefix, infix, suffix and outfix, it is decidable for NPCM languages that accept bounded languages, whether they are $\varrho$-bounded-dense. 


\section{References}

[1] J. Autebert, J. Berstel and L. Boasson, Handbook of Formal Languages (SpringerVerlag, Berlin, 1997), Berlin, ch. Context-Free Languages and Pushdown Automata.

[2] B. S. Baker and R. V. Book, Reversal-bounded multipushdown machines, Journal of Computer and System Sciences 8(3) (1974) 315-332.

[3] J. Berstel and D. Perrin, Theory of Codes (Academic Press, Orlando, 1985).

[4] E. Chiniforooshan, M. Daley, O. H. Ibarra, L. Kari and S. Seki, One-reversal counter machines and multihead automata: Revisited, Theoretical Computer Science $\mathbf{4 5 4}$ (2012) 81-87.

[5] J. Eremondi, O. Ibarra and I. McQuillan, Deletion operations on deterministic families of automata, Lecture Notes in Computer Science, eds. R. Jain, S. Jain and F. Stephan 12th Annual Conference on Theory and Applications of Models of Computation, TAMC 2015, Singapore 9076 (2015), pp. 388-399.

[6] S. Ginsburg, The Mathematical Theory of Context-Free Languages (McGraw-Hill, Inc., New York, NY, USA, 1966).

[7] S. Ginsburg and S. Greibach, Deterministic context free languages, Information and Control 9(6) (1966) 620-648.

[8] J. E. Hopcroft and J. D. Ullman, Introduction to Automata Theory, Languages, and Computation (Addison-Wesley, Reading, MA, 1979).

[9] O. Ibarra and C. Kim, A useful device for showing the solvability of some decision problems, Proceedings of the Eighth Annual ACM Symposium on Theory of Computing, STOC 'r6, (ACM, New York, NY, USA, 1976), pp. 135-140.

[10] O. Ibarra and H. Yen, On the containment and equivalence problems for two-way transducers, Theoretical Computer Science 429 (2012) 155-163.

[11] O. H. Ibarra, Reversal-bounded multicounter machines and their decision problems, Journal of the ACM 25(1) (1978) 116-133.

[12] O. H. Ibarra and S. Seki, Characterizations of bounded semilinear languages by oneway and two-way deterministic machines, International Journal of Foundations of Computer Science 23(6) (2012) 1291-1306.

[13] M. Ito, Dense and disjunctive properties of languages, Fundamentals of Computation Theory 1993, ed. Z. Ésik, Lecture Notes in Computer Science 710 (Springer Berlin Heidelberg, 1993), pp. 31-49.

[14] H. Jürgensen, L. Kari and G. Thierrin, Morphisms preserving densities, International Journal of Computer Mathematics 78 (2001) 165-189.

[15] H. Jürgensen and S. Konstantinidis, Handbook of Formal Languages (Springer-Verlag, Berlin, 1997), Berlin, ch. Codes.

[16] M. L. Minsky, Recursive unsolvability of Post's problem of "tag" and other topics in theory of Turing Machines, Annals of Mathematics 74(3) (1961) pp. 437-455.

[17] E. Post, A variant of a recursively unsolvable problem, Bulletin of the American Mathematical Society 52 (1946) 264-268.

[18] G. Sénizergues, $L(A)=L(B)$ ? decidability results from complete formal systems, Theoretical Computer Science 251(1-2) (2001) 1-166.

[19] H. J. Shyr, Free Monoids and Languages, third edn. (Hon Min Book Company, Taichung, 2001). 